\title{
Sensibilidade e Especificidade da Oximetria Fetal de Pulso e da Cardiotocografia durante o Parto: Comparação entre os Métodos no Prognóstico de Recém-Nascidos Acidóticos
}

\begin{abstract}
Sensitivity and Specificity of Fetal Pulse Oximetry and Cardiotocography during Labor: Comparison of Both Methods Regarding Prognosis of Acidotic Newborns
\end{abstract}

Edson Nunes de Morais, Patricia Spara, Francisco Maximiliano Pancich Gallarreta

\begin{abstract}
RESUMO
Objetivo: estudar a sensibilidade e a especificidade dos valores de saturação de oxigênio fetal $\left(\mathrm{SpO}_{2}\right)$ e padrões da freqüência cardiaca fetal (FCF) durante o parto, no prognóstico de fetos acidóticos ao nascimento.

Pacientes e Métodos: os valores fetais de $\mathrm{SpO}_{2}$ foram obtidos pela técnica da oximetria de pulso. Um valor de $\mathrm{SpO}_{2} \geq 30 \%$ foi considerado normal, e <30,0\%, num tempo $>10$ minutos no intervalo entre contrações, foi considerado anormal. A $\mathrm{SpO}_{2}$ fetal e os traçados de FCF foram obtidos continuamente no primeiro e segundo periodos do parto. A classificação utilizada para a FCF foi a do NICHD ${ }^{19}$.

Resultados: um total de 72 casos foram estudados. A sensibilidade e especificidade com base na $\mathrm{SpO}_{2}$ fetal foram respectivamente de 61,5\% e 96,6\%, ao passo que a sensibilidade e especificidade baseadas nos padrões de FCF foram respectivamente 69,2\% e 66,1\%. Os valores preditivos positivo e negativo em função da $\mathrm{SpO}_{2}$ fetal foram respectivamente $80 \%$ e 91,9\%; em função dos padrões de FCF foram respectivamente 31\% e 90,7\%.

Conclusões: uma boa especificidade da $\mathrm{SpO}_{2}$ para o prognóstico de recém-nascidos acidóticos foi encontrada, se comparada com a especificidade dos padrões de FCF, ao passo que a sensibilidade foi relativamente baixa para os dois métodos. Entretanto, o número de fetos acidóticos é muito pequeno para conclusões.
\end{abstract}

PALAVRAS-CHAVE: Sofrimento fetal. Trabalho de parto. Monitorização fetal.

\section{Introdução}

A oximetria de pulso é um novo método para monitorizar o feto durante o trabalho de parto. Possibilita uma melhor avaliação da condição conceptual quando utilizada em associação à cardiotocografia ${ }^{20,21}$. A monitorização eletrônica da freqüência cardiaca fetal $(\mathrm{FCF})$, desenvolvida no

Departamento de Ginecologia e Obstetrícia do Centro de Ciências da Saúde da Universidade Federal de Santa Maria Correspondência:

Edson Nunes de Morais

Av. Borges de Medeiros, 1699/604

97015-090 - Santa Maria - RS

Tel (55) 222-1942 - Fax (55) 220-8018

e-mail: edson@ccs.ufsm.br final dos anos 50 e implementada na prática clínica no início dos anos 70 , é o método mais comum de acompanhamento do feto durante o trabalho de parto ${ }^{6,10,16,17}$. Este método, que foi concebido para detectar indirtamente a hipoxemia e a acidose fetal, possui no entanto uma baixa especificidade na predição do comprometimento conceptual ao nascimento ${ }^{1}$. O padrão mais ominoso da FCF se associa no máximo com uma incidência de 50-60\% de depressão neonatal ${ }^{4}$.

A oximetria de pulso, que possibilita a informação contínua dos niveis de saturação de oxigênio arterial, é um método bem estabelecido no campo da neonatologia na monitorização de recém-nascidos de risco ${ }^{9}$. Durante os últimos anos 
esta técnica tem sido desenvolvida com o objetivo de vir a ser um possivel complemento da cardiotocografia durante o trabalho de parto ${ }^{2,12,14}$. Associada à cardiotocografia, a oximetria fetal de pulso poderá fazer uma melhor diferenciação entre os padrões de alto e de baixo risco para o feto.

O objetivo do presente trabalho foi o de estudar a saturação de oxigênio fetal durante o trabalho de parto, utilizando a técnica da oximetria fetal de pulso, e suas relações com os padrões anormais da FCF na predição de recém-nascidos acidóticos.

\section{Pacientes e Métodos}

A saturação de oxigênio fetal foi medida continuamente durante o trabalho de parto pela oximetria de pulso em 72 parturientes no Hospital Universitário de Santa Maria. A aprovação do projeto de pesquisa foi obtida previamente pelas Comissões de Ética e de Pesquisa do Centro de Ciências da Saúde da Universidade Federal de Santa Maria. Todas as pacientes assinaram o termo de consentimento esclarecido.

Os critérios de inclusão foram: gestação única de termo (idade gestacional $\geq 37$ semanas), apresentação de vértice, peso fetal ao nascimento $\geq 2.500 \mathrm{~g}$, membranas amnióticas rotas no início da monitorização com tempo inferior a 12 horas, dilatação cervical de pelo menos $2 \mathrm{~cm}$, altura da apresentação no mínimo no plano -1 de De Lee e assinatura do termo de consentimento esclarecido. Os critérios de exclusão foram: gestação múltipla, idade gestacional inferior a 37 semanas, dilatação cervical menor que $2 \mathrm{~cm}$, altura da apresentação acima do plano - 1 de De Lee, mais de uma cicatriz de cesárea prévia, placenta prévia, corioamnionite, sangramento vaginal de origem desconhecida, anomalias uterinas, doenças sexualmente transmissiveis e não-signatária do termo de consentimento esclarecido.

O peso fetal ao nascimento, o índice de Apgar e as complicações neonatais foram registradas por berçarista do Hospital Universitário. Todos os neonatos foram observados até a alta hospitalar.

$O$ registro da atividade uterina foi obtido por método externo, pela colocação de tocotransdutor na altura do corno uterino oposto ao lado do dorso fetal. A monitorização da FCF foi realizada pela técnica externa e/ou interna. Quando externa, utilizava-se transdutor de FCF (ultra-som) colocado no abdômen materno no lado do dorso fetal. Quando interna, inseria-se no couro cabeludo fetal um eletrodo em espiral de Hon tipo 1, na altura de um dos parietais do concepto. A cardiotocografia direta, interna, era realizada sempre que o registro obtido pelo método externo era de má qualidade ou os padrões da FCF mostravam-se alterados. A classificação dos padrões de FCF utilizada no estudo foi aquela recomendada pelo "National Institute of Child Health and Human Development" (NICHD) ${ }^{19}$. O cardiotocógrafo utilizado no presente estudo foi da Corometrics Medical System Inc. modelo $129 \mathrm{~F}$.

A saturação de oxigênio fetal, $\mathrm{SpO}_{2}$, foi registrada continuamente pela técnica da oximetria DE pulso. Rotas as membranas amnióticas, espontânea ou artificialmente, o toque obstétrico era realizado para o diagnóstico de variedade de posição e altura da apresentação fetal. A seguir introduzia-se o sensor descartável de oxigênio, situado entre os dedos do operador, até que a saliência tátil no cabo do sensor se colocasse na entrada do canal cervical, o que traduzia o posicionamento do corpo do equipamento na face conceptual. O registro oximétrico era iniciado apenas quando era obtida uma boa qualidade do sinal de $\mathrm{SpO}_{2}$, detectada no painel do monitor fetal $^{18}$. Considerou-se como saturação de $\mathrm{O}_{2}$ fetal dentro dos limites da normalidade quando o traçado oximétrico apresentava valores de saturação superiores a $30 \%$. Como alteração da oximetria fetal foram considerados valores inferiores a $30 \%$ de saturação de oxigênio, por um período superior a 10 minutos consecutivos entre os intervalos intercontraturais ${ }^{6}$. Para a indicação de interrupção da gestação por sofrimento fetal agudo consideraram-se apenas os niveis de $\mathrm{SpO}_{2}$ fetal ou alteração patológica da FCF quando perdida a qualidade do sinal de registro oximétrico. Para a análise da saturação de oxigênio fetal levou-se em consideração a média dos valores obtida durante o registro de $\mathrm{SpO}_{2}$ durante o primeiro e segundo periodo do parto, nos casos de saturação igual ou superior a $30 \%$. Nos casos em que a $\mathrm{SpO}_{2}$ era inferior a $30 \%$ por periodo superior a 10 minutos entre as contrações uterinas, a média era obtida apenas durante este período, arrolando-se o caso no grupo com $\mathrm{SpO}_{2}<30 \%$. O valor médio da $\mathrm{SpO}_{2}$ em cada caso era consignado pelo somatório dos pontos registrados a cada 30 segundos e dividido pelo número de total pontos. Os casos foram analisados levando-se em conta que o último registro da $\mathrm{SpO}_{2}$ deveria ocorrer no máximo até 30 minutos antes do desprendimento fetal.

$O$ equipamento utilizado neste estudo para medir a saturação de oxigênio fetal durante o trabalho de parto foi um sensor descartável da Nellcor Puritan Bennett Inc., Pleasanton, CA, modelo FS-14B.

O sangue dos vasos umbilicais era obtido por meio do pinçamento do cordão (duas pinças 
próximas ao coto umbilical e uma próxima ao intróito vaginal) antes que o recém-nascido realizasse sua primeira incursão inspiratória. Seccionado o cordão, coletavam-se amostras de sangue de artéria e veia umbilical em seringas previamente heparinizadas, em quantidades suficientes para a medida de $\mathrm{pH}$ e demais parâmetros do equilíbrio ácido-base. Considerouse como padrão bioquímico alterado um $\mathrm{pH}$ da artéria umbilical $<7,15^{22}$. $\mathrm{O} \mathrm{pH}$ e gases do sangue do cordão umbilical foram medidos em equipamentos AVL, Automatic Blood Gas System, modelos 990 e Compact 1, do Laboratório Central de Análises Clínicas do Hospital Universitário.

Para a análise dos dados, a sensibilidade (proporção de recém-nascidos corretamente classificados como acidóticos), a especificidade (proporção de recém-nascidos corretamente classificados como não-acidóticos), o valor preditivo positivo (proporção de recém-nascidos corretamente classificados como acidóticos e com padrões anormais de $\mathrm{FCF}$ ou niveis anormais de $\mathrm{SpO}_{2}$ ) e o valor preditivo negativo (proporção de recémnascidos corretamente classificados como nãoacidóticos e com padrões normais de FCF ou niveis normais de $\mathrm{SpO}_{2}$ ) foram determinados. Utilizou-se ainda o teste não-paramétrico do $\chi^{2}$ para duas amostras independentes.

\section{Resultados}

Um total de 72 casos foram estudados no Hospital Universitário de Santa Maria. A idade materna média foi de $25,1 \pm 7,3$ anos e a idade gestacional média foi de $39,2 \pm 1,6$ semanas. A paridade variou entre 1 e 9 e 32 gestantes eram nuliparas. O peso fetal médio ao nascimento foi de 3.243 gramas com limites entre 2.510 and 4.490 gramas. O tempo médio de monitorização fetal pela oxicardiotocografia foi de 151,6 $\pm 101,3$ minutos. O índice de cesárea foi de $12,5 \%$ (9 casos), sendo que o sofrimento fetal foi a indicação da cirurgia em 4 casos $\left(\mathrm{SpO}_{2}<30,0 \%\right)$. O parto instrumentado, fórcipe, ocorreu em 23,6\% das vezes (17 casos), sendo que em 6 casos por sofrimento fetal agudo. O parto vaginal espontâneo teve um indice de $63,9 \%$ (46 casos). Não foi observada nenhuma complicação materna ou do recém-nascido nos períodos puerperal e neonatal.

A sensibilidade, a especificidade, os valores positivo e negativo para o prognóstico de recémnascidos acidóticos ( $\mathrm{pH}$ de artéria umbilical $<7,15$ ), com base nos parâmetros da FCF, foram respectivamente de $69,2 \%, 66,1 \%, 31,0 \%$ e $90,7 \%$.
Observa-se que houve um bom valor preditivo negativo, sensibilidade e especificidade relativamente baixas e um valor preditivo positivo baixo (Tabela 1). A sensibilidade, a especificidade e os valores positivo e negativo para o prognóstico de recém-nascidos acidóticos ( $\mathrm{pH}$ de artéria umbilical $<7,15$ ), com base nos valores de $\mathrm{SpO}_{2}$ fetal, foram respectivamente de $61,5,96,6,80,0$ e 91,9\%. Podese dizer que houve uma boa especificidade, um bom valor preditivo negativo e razoável valor preditivo positivo. Por outro lado a sensibilidade foi relativamente baixa (Tabela 2). É provável que o número de casos possa ter algum papel nesse sentido.

Tabela 1 - Sensibilidade, especificidade, valores preditivos positivo e negativo da FCF no prognóstico de recém-nascidos acidóticos.

\begin{tabular}{lrrrcrcr}
\hline $\begin{array}{l}\text { pH da artéria } \\
\text { umbilical }\end{array}$ & \multicolumn{2}{c}{$<7,15$} & \multicolumn{2}{c}{$\geq \mathbf{7 , 1 5}$} & \multicolumn{2}{c}{ Total } \\
FCF & $\mathbf{n}$ & $\%$ & $\mathbf{n}$ & $\%$ & $\mathbf{n}$ & $\%$ \\
\hline Anormal & 9 & 31,0 & 20 & 68,9 & 29 & 40,3 \\
Normal & 5 & 9,3 & 39 & 90,7 & 43 & 59,7 \\
Total & 13 & 18,1 & 59 & 81,9 & 72 & 100,0 \\
\hline
\end{tabular}

Sensibilidade $=69,2 \%$

Valor preditivo positivo $=31,0 \%$

$\chi^{2}=4,15 p<0,05$

Tabela 2 - Sensibilidade, especificidade, valores preditivos positivo e negativo da $\mathrm{SpO}_{2}$ fetal no prognóstico de recém-nascidos acidóticos.

\begin{tabular}{|c|c|c|c|c|c|c|}
\hline pH de artéria & $<7$ & & $\geq 7$ & ,15 & & tal \\
\hline $\mathrm{SpO}_{2}$ & $n$ & $\%$ & $n$ & $\%$ & $n$ & $\%$ \\
\hline$<30 \%$ & 8 & 80,0 & 2 & 20,0 & 10 & 13,9 \\
\hline$\geq 30 \%$ & 5 & 8,1 & 57 & 91,9 & 62 & 86,1 \\
\hline Total & 13 & 18,1 & 59 & 81,9 & 72 & 100.0 \\
\hline
\end{tabular}

$\chi^{2}=25,5 p<0,0001$

Observou-se uma associação significante entre os padrões de FCF intraparto e o $\mathrm{pH}$ da artéria umbilical ( $\mathrm{p}<0,05)$, da mesma forma que uma associação altamente significante foi observada entre os valores de $\mathrm{SpO}_{2}$ fetal intraparto e o $\mathrm{pH}$ da artéria umbilical ( $\mathrm{p}<0,0001)$.

Todos os recém-nascidos com $\mathrm{SpO}_{2} \geq 30 \%$ nasceram com Apgar $\geq 7$. Dos casos com $\mathrm{SpO}_{2}$ $<30 \%$ (10 casos) em 4 casos (3 nascidos de fórcipe e 1 de cesárea) os recém-nascidos apresentaram no $1^{\circ}$ minuto Apgar $<7$; todos se recuperaram no $5^{\circ}$ minuto. 


\section{Discussão}

A medicina não é livre de subjetividade. Quando um novo método é introduzido no uso clínico, graves conseqüências podem resultar se o entusiasmo excede a habilidade de avaliar criticamente as vantagens e as desvantagens do método ${ }^{13}$. O melhor exemplo dessa afirmativa pode ser dado pela cardiotocografia. Este método, introduzido na prática clínica no final dos anos 60 e início dos 70 , não foi adequadamente avaliado antes de ser largamente utilizado ${ }^{20}$.

O principal objetivo da oximetria fetal de pulso, quando utilizada como método adjunto da monitorização fetal eletrônica, é o de melhorar a especificidade da avaliação conceptual. No entanto, todo estudo utilizando este novo método deve ser judiciosamente analisado para que não se incorra nos equívocos cometidos com a cardiotocografia que, apesar de ser um excelente método de avaliação conceptual intraparto, foi infelizmente colocada sob suspeita por grande parte dos investigadores, até que ensaios clínicos controlados randomizados a colocaram no devido lugar.

No presente estudo buscou-se relacionar os niveis de $\mathrm{SpO}_{2}$ fetal com os padrões de $\mathrm{FCF}$, em função do $\mathrm{pH}$ da artéria umbilical. Levaram-se em consideração padrões de normalidade de saturação de oxigênio $>30 \%$, conforme descrito por diferentes autores $^{11,13}$.

Estudos buscando estabelecer os niveis de normalidade da $\mathrm{SpO}_{2}$ durante o trabalho de parto têm sido realizados. No entanto, os valores encontrados pelos diversos autores têm variado numa faixa bastante ampla. Por outro lado, os pesquisadores concordam que niveis superiores a $30 \%$ de saturação de oxigênio fetal associam-se com recém-nascidos em boas condições de vitalidade. Em 1994, Dildy et al. ${ }^{8}$, estudando 160 casos de fetos considerados normais, relatam niveis de $59 \pm 10,0 \%$ e $53 \% \pm 10,0 \%$ respectivamente para o $1^{\circ}$ e $2^{\circ}$ períodos do parto, ao passo que Morais et al. ${ }^{15}$ encontraram valores médios de $\mathrm{SpO}_{2}$ de $53 \pm 7,3 \%$ e $46,8 \pm 7,7 \%$ respectivamente para o primeiro e segundo periodo do parto, quando o $\mathrm{pH}$ do sangue da artéria umbilical era $>7,20$. Já Di Renzo et al. ${ }^{5}$ observaram valores de $\mathrm{SpO}_{2}$ fetal de $44 \%$ para o primeiro período e $47 \%$ para o segundo período.

Qualquer estudo que objetive relacionar a saturação de oxigênio fetal com padrões de FCF pode ter como fator limitante a interpretabilidade de tais padrões. A recente recomendação do "National Institute of Child Health and Human Development" (NICHD) ${ }^{19}$ tem tido boa aceitação pelos diferentes autores, embora alguns itens da classificação proposta mereçam criticas pertinentes $^{3}$. Os padrões de FCF analisados neste estudo obedeceram à classificação proposta pelo NICHD. Desta forma, a sensibilidade e a especificidade da FCF para o prognóstico de recémnascidos acidóticos, em função do $\mathrm{pH}$ da artéria umbilical, foram estabelecidas.

Um estudo multicêntrico realizado por van den Berg et al. ${ }^{22}$, na qual quatro obstetras experts individualmente avaliaram 119 casos monitorizados durante o trabalho de parto, mostrou que a sensibilidade baseada somente na cardiotocografia variou entre $43 \%$ e $100 \%$ e a especificidade entre $54 \%$ e $88 \%$ entre os quatro avaliadores. Quando a $\mathrm{SpO}_{2}$ foi adicionada à cardiotocografia a sensibilidade variou de zero a $86 \%$ e a especificidade de $75 \%$ a $93 \%$. Butterwegge ${ }^{2}$ demonstrou que um padrão não-seguro da $\mathrm{FCF}$ e uma $\mathrm{SpO}_{2}$ estável acima de $40 \%$ refletiram um bemestar fetal em que o $\mathrm{pH}$ da artéria umbilical era $>7,20$ e com bons resultados neonatais.

Este estudo mostrou uma boa especificidade da $\mathrm{SpO}_{2}$ para fetos acidóticos ao nascimento $(\mathrm{pH}$ de artéria umbilical $<7,15$ ) se comparada com os padrões da FCF $(96,6 \%$ vs $66,1 \%)$. Por outro lado, a sensibilidade mostrou-se semelhante para os dois métodos estudados, $61,5 \%$ para a $\mathrm{SpO}_{2}$ e $69,2 \%$ para a FCF. A probabilidade de um feto nascer acidótico quando a $\mathrm{SpO}_{2}$ fetal mostrava niveis inferiores a 30\% foi de $80 \%$, bem superior à FCF (31\%).

Ainda que os resultados do presente estudo se assemelhem aos de outros autores, para algumas proporções estudadas, nós não podemos concluir se a associação dos dois métodos melhora a predição de um recém-nascido comprometido, pois o número de fetos acidóticos do presente estudo é muito pequeno (10 casos). Serão necessárias novas pesquisas com amostras mais numerosas para que se possa tirar conclusões definitivas. O estabelecimento de pontos de corte de valores de $\mathrm{pH}$ do sangue da artéria umbilical, em função da $\mathrm{SpO}_{2}$ fetal obtida o mais próximo do nascimento é, sob o nosso ponto de vista, fundamental.

$\mathrm{O}$ avanço tecnológico na monitorização do equilíbrio ácido-base revolucionou a prática da medicina em campos como o da anestesia e dos cuidados intensivos de adultos e de recém-nascidos. Os obstetras não vêem uma mudança significativa na avaliação do bem-estar fetal intraparto desde a introdução da monitorização da FCF e do estudo bioquímico de amostras de sangue capilar do couro cabeludo fetal, isto é, há quase 40 anos $^{7}$.

A oximetria fetal de pulso tem um potencial para melhorar o uso da informação sobre as condições fetais durante o trabalho de parto, e a monitorização da $\mathrm{SpO}_{2}$ pode e deve ser avaliada por meio de estudos controlados randomizados, preferencialmente multicêntricos, antes que possa ser usada na prática clínica de rotina ${ }^{8}$. 
SUMMARY

Purpose: to study the sensitivity and specificity based on fetal oxygen saturation ( $\mathrm{SpO}$ ) values and fetal heart rate (FHR) patterns during labor, for the prognosis of acidotic fetuses at birth.

Patients and Methods: $\mathrm{SpO}$, values were obtained by fetal pulse oximetry technique. A fetal $\mathrm{SpO}_{2}$ value $\geq 30 \%$ was considered normal, and an $\mathrm{SpO}_{2}$ which remained $<30.0 \%$ for more than 10 min between contractions was considered abnormal. Fetal $\mathrm{SpO}_{2}$ and FHR tracings were obtained during the first and second stage of labor. FHR classification used in the study has been derived from the National Institute of Child Health and Human Development ${ }^{19}$.

Results: a total of 72 subjects were studied. The sensitivity and specificity, based on $\mathrm{SpO}_{2}$, were $61.5 \%$ and $96.6 \%$, respectively, whereas the sensitivity and specificity based on FHR patterns were $69.2 \%$ and $66.1 \%$. Positive and negative predictive values based on $\mathrm{SpO}_{2}$ were $80 \%$ and $91.9 \%$, respectively, and based on FHR patterns were $31 \%$ and $90.7 \%$, respectively.

Conclusions: a good fetal $\mathrm{SpO}_{2}$ specificity for prognosis of acidotic fetuses at birth was found, if compared with FHR pattern specificity, whereas sensitivity was poor for both methods. However, the number of acidotic fetuses was too small to allow more conclusions.

KEY WORDS: Fetal distress. Labor. Fetal cardiotocography.

\section{Referências}

1. Banta HD, Thacker SB. Assessing the costs and benefits of electronic fetal monitoring. Obstet Gynecol Surv 1979;34: 627-42.

2. Butterwegge M. Fetal pulse oximetry and nonreassuring heart rate. Eur J Obstet Gynecol Reprod Biol 1997;72 (Suppl. 1): S63-S66.

3. Cibils LA. Interpretation of intrapartum fetal heart rate tracings. Am J Obstet Gynecol (Letter) 1998;179:1099-100.

4. Clark SL, Gimovsky ML, Miller FC. The scalp stimulation test: A clinical alternative to fetal scalp blood sampling. Am J Obstet Gynecol 1984;148:274-7.

5. Di Renzo GC, Cenci F, Saab JC, Ferrari G, Clerici G. Pulse oximetry: An additional fetal monitoring during labor. 2nd World Congress on Labor \& Delivery, May 6-9, 1997, Rome, Italy.

6. Dildy GA, Clark SL, Garite TJ, Porter TF, Swedlow DB, Varner MW. Current status of the multicenter randomized clinical trial on fetal oxygen saturation monitoring in the United States. Eur J Obstet Gynecol Reprod Biol 1997; 72 (Suppl. 1): S43-S50.

7. Dildy GA, Clark SL, Loucks CA. Fetal pulse oximetry: Past, present, and future. Am J Obstet Gynecol 1996;175:1-9.

8. Dildy GA, van den Berg PP, Katz M, Clark SL, Jongsma HW, Nijhuis JG, Loucks CA. Intrapartum fetal oximetry: Fetal oxygen saturation trends during labor and relation to delivery outcome. Am J Obstet Gynecol 1994;171:679-84.

9. Franconi S. Reliability of pulse oximetry in hypoxic infants. J Pediatr 1988;112:424-7.

10. Hon EH. The electrical evaluation of the fetal heart rate. Am J Obstet Gynecol 1958;75: 1215-9.

11. Kuhnert M, Seelbach-Goebel B, Butterwegge M. Predicting the fetal arterial oxygen saturation and fetal scalp pH: Results of the German multicenter study. Am J Obstet Gynecol 1998;178:330-5.

12. Langer B, Carbonne B, Goffinet F, Le Gouëff F, Berkane N, Laville M. Fetal pulse oximetry and fetal heart rate monitoring during stage II of labour. Eur J Obstet Gynecol Reprod Biol 1997;72 (Suppl. 1): S57-S61.

13. Maesel A, Martensson L, Gudmundsson S, Marsal K. Fetal pulse oximetry. A methological study. Acta Obstet Gynecol Scand 1996;75:144-8.

14. McNamara E, Johnson N. Fetal monitoring by pulse oximetry and CTG. J Perinat Med 1994;22:475-80.

15. Morais EN, Gallarreta MPM, Spara P. Saturação de oxigênio fetal medida pela oximetria fetal de pulso durante o trabalho de parto: Relações com o pH de artéria umbilical. Rev Bras Ginecol Obstet 1999; 21:141-6.

16. Morais EN, Sokol MG, Mottecy CS. Valoración de la taquicardia como un signo de sufrimiento fetal intraparto. Acta Obstet Ginecol Hisp Lusit 1983;31:489-98.

17. Morais EN, Spara P, Farias FM. Repercussão da monitorização fetal intraparto sobre os índices de operação cesariana. Rev Bras Ginecol Obstet 1998;20:77-81.

18. Morais EN. Princípios e técnica da oximetria fetal de pulso durante o trabalho de parto. Femina 1998;26:561-4.

19. National Institute of Child Health and Human Development (NICHD). Research Planning Workshop: Electronic fetal heart rate monitoring. Research guidlines for interpretation. Am J Obstet Gynecol 1997;177:1385-90.

20. Saling E. Fetal pulse oximetry during labor: Issues and recommendations for clinical use. J Perinat Med 1996;24:467-78.

21.Seelbach-Goebel B, Butterwegge M, Kuhnert M, Heupel M. Fetale Reflexionspulsoxymetrie sub partu. Erfahrungen - prognostische Bedeutung und Konsequenz - Ziele. $Z$ Geburtshilfe Perinatol 1994;198:67-71.

22. van den Berg, Dildy GA, Luttkus A, Mason GC, Harvey CJ, Nijhuis JG, Jongsma HW. The efficacy of intrapartum fetal surveillance when fetal pulse oximetry is added to cardiotocography. Eur J Obstet Gynecol Reprod Biol 1997;72 (Suppl. 1): S67-S71. 